\title{
Risk Faktörü Olmadan Oluşan Bilateral Spontan Aşil Tendon Rüptürü
}

\section{Bilateral Spontaneous Rupture of Achilles Tendons In Absence of Risk Factors}

Faik Türkmen ${ }^{1}$,

Mustafa Özer ${ }^{1}$,

Burkay Kutluhan Kaçıra ${ }^{1}$,

İsmail Hakkı Korucu ${ }^{1}$,

Gani Göncü ${ }^{1}$

${ }^{1}$ Necmettin Erbakan Üniversitesi Meram Tıp Fakültesi Ortopedi ve Travmatoloji Anabilim Dalı, Konya, Türkiye.

Geliş Tarihi/Received: 25 Ocak 2017 Kabul Tarihi/Accepted: 9 Mayıs 2018

Yazışma Adresi: Faik Türkmen,

Necmettin Erbakan Üniversitesi Meram Tıp Fakültesi Ortopedi ve Travmatoloji Anabilim Dalı, Konya, Türkiye

e-posta: turkmenfaik@gmail.com

ORCID

Faik Türkmen

https://orcid.org/0000-0002-9293-645X

\begin{abstract}
Öz
Aşil tendon rüptürleri yaygın kas-iskelet sistemi yaralanmalarıdır. Genellikle tek tarafıı olarak aktif orta yas bireylerde meydana gelir. Herhangi bir yatkınlık oluşturan risk faktörü olmadan oluşan spontan rüptürler nadirdir ve bunun da bilateral olması oldukça nadirdir. Biz, herhangi bir risk faktörü olmayan spontan bilateral aşil tendon rüptürü olan 32 yaşındaki erkek olguyu sunuyoruz. Rüptür halter kaldırma sonrası ağırlığa bağlı olarak bilateral gastroknemius kompleksinde meydana gelen aşırı stres ve uzamaya bağlı olarak meydana gelmiști. Klinik tanı konduktan sonra ultrasonografi ile bilateral rüptür gösterildi. Hastaya bilateral cerrahi tamir uygulandı. Rehabilitasyon 6 hafta sonra başlandı. Ameliyattan 8 hafta sonra kısmi, 12 hafta sonra tam yük vererek mobilize olan hasta yaralanma öncesi iş ve sosyal yaşantısına sorunsuz döndü. Bilateral aşil tendon rüptürleri çok nadirdir fakat yüksek farkındalık karşı taraf rüptürlerinin gözden kaçmasını önleyebilecektir
\end{abstract}

Anahtar Kelimeler: Aşil tendon, rüptür/cerrahi, bilateral, spontan.

\section{Abstract}

Achilles tendon rupture is a common musculoskeletal injury. It usually occurs unilaterally in active middleaged individuals. Spontaneous ruptures without any risk factors predisposing the patient is uncommon and for it to occur bilaterally is very uncommon. We report a case of bilateral spontaneous Achilles tendon rupture in a 32-year-old man with no certain previous and current risk factors. The ruptures occurred after lifting barbell in which the weight caused a severe stress and elongation of the bilaterally gastrocnemius complex. After a clinical examination confirmed the diagnosis, ultrasonography of the Achilles tendons revealed bilateral ruptures. The patient underwent bilateral primary surgical repair. Rehabilitation was begun 6 weeks later. We allowed the weightbearing partially at 8 weeks after surgery, fully at 12 weeks after surgery. The patient's return to premorbid work and social life was uneventful. Bilateral Achilles tendon ruptures are very rare but increased awareness would help avoid a rupture of the contralateral side being missed.

Keywords: Achilles tendon, rupture/surgery, bilateral, spontaneous.

\section{GiRiş}

Aşil tendon, vücudun en kalın ve en güçlü tendonudur. Vücut ağırlığının 12 katına kadar kuvvetlere dayanabilir ancak alt ekstremitenin en çok rüptüre olan tendonudur (1). Aşil tendon rüptürü sıklıkla, travma ve zıplama gibi atletik aktiviteler sonrası oluşur ve genellikle tek taraflıdır (2). Yaralanma mekanizması dorsifleksiyonda ayak üzerine, gastroknemius ve soleus kaslarındaki ani ve güçlü kasılmayı içerir (yüksek enerjili hareketlerde eksantrik yüklenme ile) ve genellikle orta yaş (30-50) erkekleri etkiler (3). Genel popülasyonda aşil tendon rüptürü insidansı $\% 0,02$ iken, bunların $\% 1$ 'inden daha azı bilateraldir $(4,5)$. Spontan bilateral aşil tendon rüptürü için risk faktörleri; kortikosteroid kullanımı, anabolik steroidler, fluorokinolonlar, diyabet, kronik böbrek yetmezliği, daha önceki aşil tendon rüptürü ve kronik tendinopatidir $(6,7)$. Risk faktörü olmadan oluşan spontan bilateral aşil tendon rüptürleri oldukça nadirdir $(4,5)$. Bu olgu sunumunda bilinen risk faktörü olmayan genç erkek hastada spontan bilateral aşil tendon rüptürü sunulmuştur.

\section{OLGU}

32 yaşında normal beden kitle indeksli masa başı iş yapan erkek hasta halter kaldırma sonrası her iki ayak bileği arkasında ani başlayan ağrı, ses gelmesi ve parmak uçlarında yükselememe şikayeti ile kliniğimize başvurdu. Daha önce bilinen ve tanısı konulan herhangi bir hastalığı bulunmayan ve ameliyat öncesi ve sonrası tetkiklerinde de risk faktörü bulunmayan hastanın fizik muayenesinde aşil tendonunda palpasyonla hassasiyet, aşil tendon trasesinde gap ve Thompson testi pozitif olarak

Atıf yapmak için: Türkmen F, Özer M, Kaçıra BK, Korucu İH, Göncü G. Risk Faktörü Olmadan Oluşan Bilateral Spontan Aşiltendon Rüptürü. Selcuk Med J 2019;35(3): 203-206 


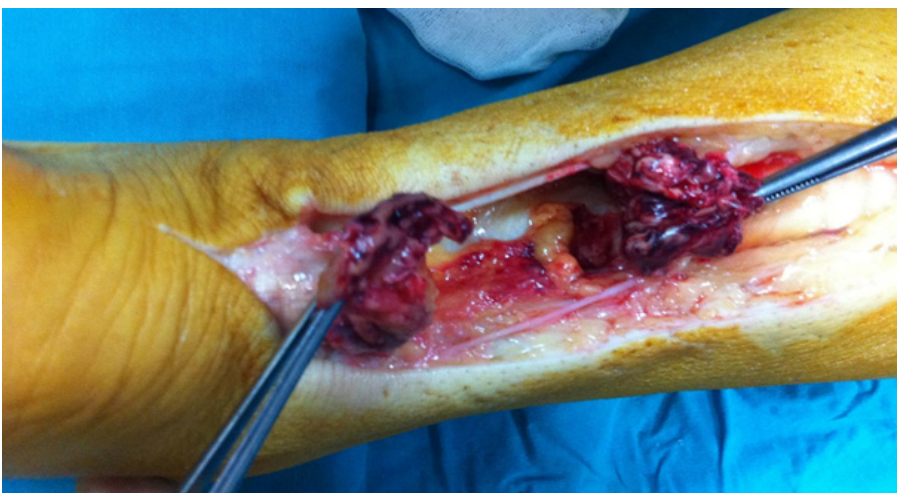

Şekil 1. Sol aşil tendom rüptür klinik görüntüsü

saptandı. Klinik tanı konduktan sonra ultrasonografi ile bilateral aşil tendon rüptürü tanısı teyit edildi ve hastaya cerrahi tedavi planlandı. Aynı seansta prone pozisyonda yatan hastanın aşil tendonu üzerinden posteromedial insizyonla girilerek aşil tendonuna ulaşıldı (Şekil 1, 2). Uçlar tazelenerek primer tamir; 5 numara emilmeyen core sütürler ve 2/0 örgülü emilebilen sütürler (çevre dikişleri) ile yapıldı. Cilt altı-cilt uygun şekilde kapatılarak, ayak plantar fleksiyonda olacak şekilde uzun bacak sirküler alçıya alındı (Şekil 3). Tromboembolitik profilaksi başlandı. 4.hafta sonrası kısa bacak alçıya geçildi. 6.haftada alçı çıkarılarak ayakbileği-ayak ortezine geçildi ve rehabilitasyona başlandı. Ameliyattan 8 hafta sonra kısmi, 12 hafta sonra tam yük vererek mobilize olan hastanın sağ tarafında 16 .haftada sütür reaksiyonu benzeri inflamasyon meydana geldi, yüzeyel debridman yapılarak çevre sütürleri alındı. Sonrasında herhangi bir sorunu olmayan hastanın 6 . ay takibinde yaralanma öncesi iş ve sosyal yaşantısına sorunsuz döndüğü görüldü.

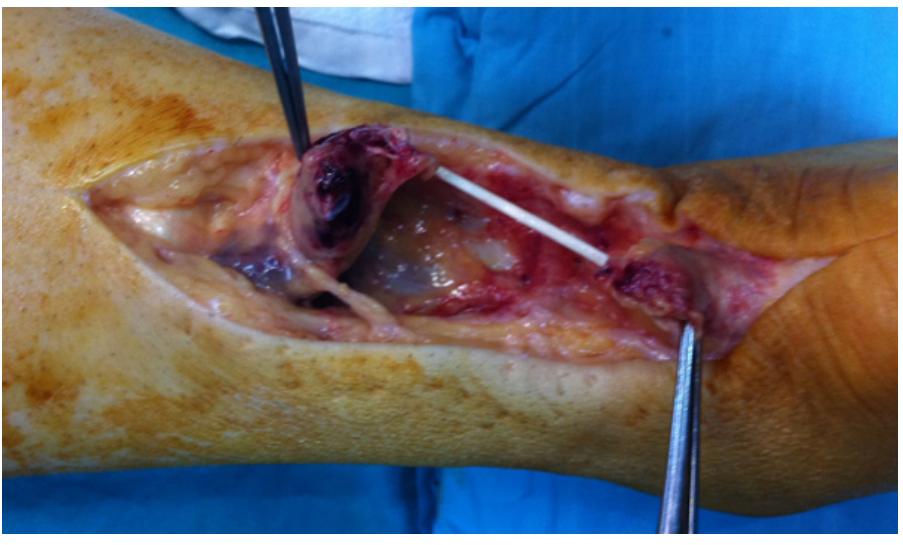

Şekil 2. Sağ aşil tendom rüptür klinik görüntüsü

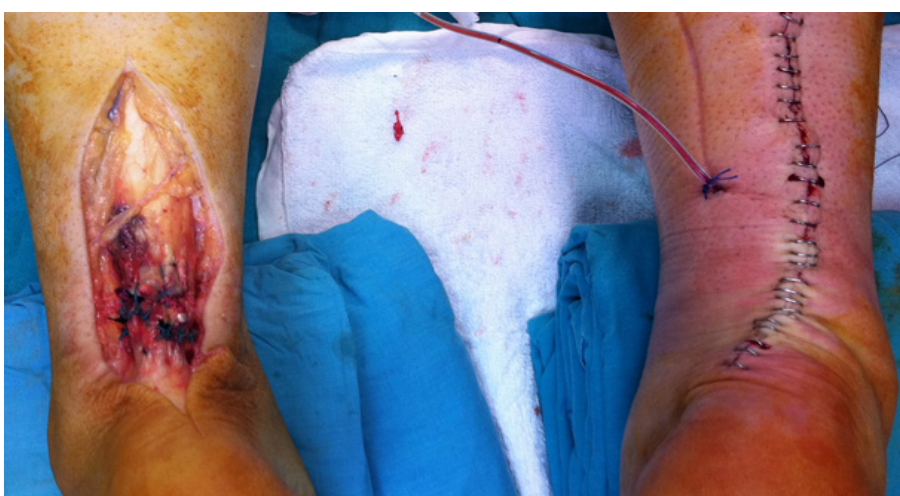

Şekil 3. Cerrahi sonrası klinik görüntü

\section{TARTIŞMA}

Aşil tendon rüptürleri sıklıkla, yaş ile beraber kanlanmanın zayıfladığı tendonun kalkaneusa yapışma yerinden 2-6 cm proksimalden olmaktadır. $(8,9)$ Aşil tendon rüptürlerinin $\% 1$ den azının bilateral olduğu ve risk faktörü olmaksızın rüptürlerin daha nadir olduğu bildirilmektedir (10). Tendon rüptürleri birçok faktöre bağlı olabilmekle beraber spontan rüptürlerin etyolojisi henüz aydınlatılabilmiş değildir (10). Bu yaralanmaya sebep olabilecek risk faktörleri açısından hastamızı değerlendirdiğimizde herhangi bir risk faktörü bulamadık. Rüptürün; halter kaldırma sonrası ağırlık nedeniyle bilateral gastroknemius kompleksinde meydana gelen aşırı stres ve uzamaya bağlı olarak meydana gelmiş olabileceğini düşündük. Literatüre baktığımızda; kronik steroid kullanımı, lokal kortikosteroid enjeksiyonu, flourokinolonların kullanımı, romatoid artrit, gut, kronik böbrek yetmezliği, diyabet ve sistemil lupus eritematozis risk faktörleri olarak belirtilmektedir $(6,7)$. Literatürde tanımlanmış rüptüre yatkınlık yapabilecek ek intrinsik nedenler ise; ayağın hiperpronasyonu, bacak uzunluk farkı ve tensil kuvvetlerin aşırı ve dengesiz dağıldığı obezitedir (11). Propriosepsiyon duyu dağılımındaki anormalliğe bağlı aşil tendon mekanik yetmezliği ile sonuçlanan rüptürler meydana gelebilmektedir. Yapılan bir çalışmada, tek taraflı aşil tendon rüptürü olan hastaların her iki ayak bileği eklemi proprisepsiyon duyularının anormal olduğu saptanmış. Buna göre aşil tendon rüptürü olan bireylerde propriosepsiyon duyusunun doğuştan yetersiz olabileceği ve bunun da mekanik yetmezlikle ilişkili olabileceği bildirilmiştir (12).

Aşil tendon rüptürleri konservatif ve cerrahi olarak tedavi edilebilmektedir. Bilateral rüptürlerde cerrahi tedavi ön plana çıkmakla birlikte konservatif tedavi edilmiş bilateral rüptür olguları bildirilmiştir (10). 
Konservatif tedavi cerrahi tedaviye göre daha fazla yeniden rüptür oranına sahiptir. Açık cerrahi tedavide yara yeri problemleri nedeniyle perkütan yöntemler geliştirilmiştir ancak perkütan yöntemlerle de süral sinir yaralanma riski ve açık cerrahi den daha fazla yeniden rüptür oranları bildirilmektedir (13). Açık cerrahide orta hat insizyonuna göre medial veya lateral insizyon tendon yapışıklığı ve skar rahatsızlığı açısından daha avantajlıdır. Primer tendon onarımında Kessler, Bunnel ve Krackow teknikleri uygulanabilirken, Bunnel ve Krackow yöntemi Kessler'e göre daha kuvvetli tutunma sağlar. Kronik ya da inmal edilmiş rüptürler sonrası augmentasyonlu tamir teknikleri kullanılabilir. $\mathrm{Bu}$ amaçla plantaris, peroneus brevis, fleksör hallusis longus ve fleksor digitorum longus tendonları ile gastrosoleus flepleri, dakron bantlarla güçlendirme yapılabilir (14). Literatürde bu sık uygulanan tedavi yöntemlerine ek yöntemler ve tendon iyileşmesi üzerine çalışmalar da vardır $(15,16)$. Bir çalışmada tek taraflı aşil tendon rüptürü cerrahi tedavisi sonrası 15 yıllık takipte dinamik pedobarografi ile cerrahi uygulanan tarafta sağlam tarafa göre daha az total temas alanı, daha yüksek basınç değerleri ve düşük bir ark indeksi var olduğu, basınç merkezinin daha lateralde olduğu ve medial arkın daha yüksek olduğu tespit edilmiş (17). Bu olguda bilateral aynı seansta açık primer onarım uygulandı. Olgumuzda tek tarafında çevresel sütürlere reaksiyon olarak geliştiği düşünülen yara yeri komplikasyonu oluştu. Yapılan yüzeyel debridman ile tedavi edildi. Aşil tendonunun kendi kanlanmasının zayıf olduğu gibi üzerini kaplayan cildin de kanlanması zayıf olduğundan açık cerrahiler sonrası yara yeri sorunları oluşabilmektedir. Bu nedenle cilt diseksiyonu ve kapatılması sırasında cilt kanlanmasını bozacak ekartasyonlardan ve cildi rahatsız edebilecek çevresel ve ciltaltı sütürlerden kaçınımalıdır (14). Akut aşil tendon rüptürlerinin hem konservatif hem de cerrahi tedavisi sonrası, konvansiyonel veya hızlandırılmış fonksiyonel rehabilitasyonlarıyla bildirilmiş komplikasyonlar vardır $(18,19)$. Biz hastamızda bilateral yaralanma olduğu için agresif bir rehabilitasyon tercih etmedik ve 12 . Haftadan sonra tam yük verdirerek mobilize ettik.

Sonuç olarak, aşil tendon rüptürleri çoğunlukla risk faktörleri bulunan kişilerde görülmektedir. Bilateral olgular daha nadir olmakla birlikte risk faktörü olmayanlarda çok daha nadir görülmektedir fakat yüksek farkındalık karşı taraf rüptürlerinin gözden kaçmasını önleyebilecektir.

Çıkar Çatışması: Çalışmada herhangi bir çıkar çatışması yoktur.
Finansal Çıkar Çatışması: Çalışmada herhangi bir finansal çıkar çatışması yoktur.

Yazışma Adresi: Faik Türkmen, Necmettin Erbakan Üniversitesi Meram Tıp Fakültesi Ortopedi ve Travmatoloji Anabilim Dalı, Konya, Türkiye

Tel: $05333841204 \quad 03322236230$

e-posta: turkmenfaik@gmail.com

\section{KAYNAKLAR}

1. Maffulli N, Kenward MG, Testa V, et al. Clinical diagnosis of achilles tendinopathy with tendinosis. Clin J Sport Med 2003;13:11-5.

2. Paavola M, Orava S, Leppilahti J, et al. Chronic achilles tendon overuse injury: Complications after surgical treatment. An analysis of 432 consecutive patients. Am J Sports Med 2000;28:77-82.

3. Jozsa L, Kvist M, Balint BJ, et al. The role of recreational sport activity in achilles tendon rupture. A clinical, pathoanatomical, and sociological study of 292 cases. Am J Sports Med 1989;17:338-43.

4. Zietek P, Karaczun M, Kruk B, et al. Minimally invasive repair of traumatic and simultaneous rupture of both achilles tendons. J Foot Ankle Surg 2016;55(3):642-4.

5. Guelfi M, Pantalone A, Vanni D, et al. Bilateral traumatic rupture of achilles tendons in absence of risk factors treated with percutaneous technique and platelet-rich plasma. J Foot Ankle Surg 2014;20:43-6.

6. Mahler F, Fritschy D. Partial and complete ruptures of the achilles tendon and local corticosteroid injections. Br J Sports Med 1992;26:7-14.

7. Doyle HE. Tendinopathy resulting from the use of fluoroquinolones: Managing risks. JAAPA 2010;23(12):1821.

8. Lagergren C, Lindholm A. Vascular distribution in the Achilles tendon: An angiographic and microangiographic study. Acta Chir Scand 1959;116:491-5.

9. Oktem H, Calgüner E, Erdoğan D, et al. Age-related changes in light microscopy with sirius red technique in rat achilles tendon. Eklem Hastalik Cerrahisi 2010;21:50-5.

10. Hashim Z, Dahabreh Z, Bin Jemain MT, et al. Bilateral simultaneous achilles tendon rupture in the absence of risk factors: A case report. Foot Ankle Spec 2012;5(1):68-72.

11. Hess GW. Achilles tendon rupture: A review of etiology, population, anatomy, risk factors, and injury prevention. Foot Ankle Spec 2010;3:29-32.

12. Bressel E, Larsen BT, McNair PJ, et al. Ankle joint proprioception and passive mechanical properties of the calf muscles after an achilles tendon rupture: A comparison with matched controls. Clin Biomech Bristol Avon 2004;19(3):28491.

13. Henriquez $H$, Munoz R, Carcuro $G$, et al. Is percutaneous repair better than open repair in acute achilles tendon rupture? Clin Orthop 2012;470(4):998-1003.

14. Rosenzweig S, Azar FM. Open repair of acute achilles tendon ruptures. Foot Ankle Clin 2009;14(4):699-709.

15. Solakoğlu $C$, Mahiroğulları $M$, et al. Fibrin sealant in the treatment of acute ruptures of the Achilles tendon: Long-term results. Eklem Hastalik Cerrahisi 2010;21:124-9.

16. Taşkesen A, Ataoğlu B, Özer $M$, et al. Glucosaminechondroitin sulphate accelerates tendon-to-bonehealing in rabbits. Eklem Hastalık Cerrahisi 2015;26(2):77-83. 
17. Atik OS, Bölükbaşı $S$, Kanatı K. Does surgical repair of acute achilles tendon rupture cause structural changes on 15-year follow-up? Eklem Hastalık Cerrahisi 2011;22(1):54-5.

18. Willits K, Amendola A, Bryant D, et al. Operative versus nonoperative treatment of acute achilles tendon ruptures: A multicenter randomized trial using accelerated functional rehabilitation. J Bone Joint Surg [Am] 2010;92:2767-75.
19. Atik OS, Tan J, Güler N. Agressive functional rehabilitation after surgical treatment of acute rupture of the Achilles tendon. Eklem Hastalik Cerrahisi 1998;2:149-50. 